\title{
Large-sphere and small-sphere limits of the Brown-York mass
}

\author{
Xu-Qian Fan, Yuguang Shi and Luen-Fai Tam
}

\begin{abstract}
In this paper, we will study the limiting behavior of the BrownYork mass of the coordinate spheres in an asymptotically flat manifold. Limiting behaviors of volumes of regions related to coordinate spheres are also obtained, including a discussion on the isoperimetric mass introduced by Huisken [14]. We will also study expansions of the Brown-York mass and the Hawking mass of geodesic spheres with center at a fixed point $p$ of a 3 -manifold. Some geometric consequences will be derived.
\end{abstract}

\section{Introduction}

In this work, we will discuss the large-sphere limit of the Brown-York mass in an asymptotically flat (AF) manifold and the small-sphere limit of the Brown-York mass near a point in a three-dimensional manifold. We will also discuss the behaviors of large-sphere limit and small-sphere limit of other interesting quantities.

Let us first recall some definitions. In general relativity, AF manifolds have great interests in many problems. In this paper, we adopt the following definition of AF manifolds.

Definition 1.1. A complete 3-manifold $(M, g)$ is said to be AF of order $\tau$ (with one end) if there is a compact subset $K$ such that $M \backslash K$ is diffeomorphic to $\mathbb{R}^{3} \backslash B_{R}(0)$ for some $R>0$ and in the standard coordinates in $\mathbb{R}^{3}$, the metric $g$ satisfies:

$$
g_{i j}=\delta_{i j}+\sigma_{i j}
$$

with

$$
\left|\sigma_{i j}\right|+r\left|\partial \sigma_{i j}\right|+r^{2}\left|\partial \partial \sigma_{i j}\right|+r^{3}\left|\partial \partial \partial \sigma_{i j}\right|=O\left(r^{-\tau}\right)
$$

for some constant $1 \geq \tau>\frac{1}{2}$, where $r$ and $\partial$ denote the Euclidean distance and standard derivative operator on $\mathbb{R}^{3}$, respectively. 
A coordinate system of $M$ near infinity so that the metric tensor in these coordinates satisfies the decay conditions in the definition is said to be admissible. Note that some of the results in the following do not need decays of the third-order derivatives of $\sigma_{i j}$.

Definition 1.2. The Arnowitt-Deser-Misner (ADM) mass (see [1]) of an AF manifold $M$ is defined as:

$$
m_{\mathrm{ADM}}(M)=\lim _{r \rightarrow \infty} \frac{1}{16 \pi} \int_{S_{r}}\left(g_{i j, i}-g_{i i, j}\right) \nu^{j} d \Sigma_{r}^{0},
$$

where $S_{r}$ is the Euclidean sphere with Euclidean radius $r$ and center at the origin, $d \Sigma_{r}^{0}$ is the volume element induced by the Euclidean metric, $\nu$ is the outward unit normal of $S_{r}$ in $\mathbb{R}^{3}$ and the derivative is the ordinary partial derivative.

We always assume that the scalar curvature is in $L^{1}(M)$ so that the limit exists in the definition. Under the decay conditions in the definition of AF manifold, the definition of ADM mass is independent of the choice of admissible coordinates by the result of Bartnik [2].

Let $(\Omega, g)$ be a compact 3 -manifold with smooth boundary $\partial \Omega$. Suppose the Gauss curvature of $\partial \Omega$ is positive, then the Brown-York quasi-local mass of $\partial \Omega$ is defined as (see $[6,7])$ :

\section{Definition 1.3.}

$$
m_{\mathrm{BY}}(\partial \Omega)=\frac{1}{8 \pi} \int_{\partial \Omega}\left(H_{0}-H\right) d \Sigma,
$$

where $H$ is the mean curvature of $\partial \Omega$ with respect to the outward unit normal and the metric $g, d \Sigma$ is the volume element induced on $\partial \Omega$ by $g$ and $H_{0}$ is the mean curvature of $\partial \Omega$ with respect to the outward unit normal when embedded in $\mathbb{R}^{3}$.

The Brown-York mass is well-defined because by the result of Nirenberg [18], $\partial \Omega$ can be isometrically embedded in $\mathbb{R}^{3}$ and the embedding is unique by Cohn-Vossen [8] (see also $[13,19,20]$ ). In particular, $H_{0}$ is completely determined by the metric on $\partial \Omega$. However, this is a global property. In contrast, the norm of the mean curvature vector of an embedding of $\partial \Omega$ into the light cone in the Minkowski space can be expressed explicitly in terms of the Gauss curvature, see [5]. Hence, in the study of Brown-York mass, one of the difficulties is to estimate $\int_{\partial \Omega} H_{0} d \Sigma$. We will use the Minkowski formulae [16] and the estimates of Nirenberg [18] in this regard. 
In the first part of this paper, we want to study limiting behaviors of Brown-York mass on large spheres. We will verify the following:

Theorem 1.1. Let $(M, g)$ be an AF manifold of order $\tau>\frac{1}{2}$ with one end and let $S_{r}$ be the coordinate spheres in some admissible coordinates. Then

$$
\lim _{r \rightarrow \infty} m_{\mathrm{BY}}\left(S_{r}\right)=m_{\mathrm{ADM}}(M)
$$

Here $m_{\mathrm{BY}}\left(S_{r}\right)$ is the Brown-York quasi-local mass of $S_{r}$, and $m_{\mathrm{ADM}}(M)$ is the ADM mass of $M$.

Theorem 1.1 was observed and proved to be true by many people, see the works of Brown-York [7], Hawking-Horowitz [12], Braden-Brown-WhitingYork [4] and Baskaran-Lau-Petrov [3], see also [23]. However, in this paper, we will use a different method to derive Theorem 1.1. Interestingly, our method leads to the following volume comparison result. Let $V(r)$ be the volume with respect to an AF metric $g$ of the region inside $S_{r}$ and let $V_{0}(r)$ be the Euclidean volume inside the surface $S_{r}$ when embedded in $\mathbb{R}^{3}$.

Theorem 1.2. Let $(M, g)$ be an AF manifold of order $\tau>\frac{1}{2}$ with one end. Then

$$
V_{0}(r)-V(r)=-2 m_{\mathrm{ADM}}(M) \pi r^{2}+o\left(r^{2}\right) .
$$

Hence if the ADM mass is non-negative, then $\lim _{r \rightarrow \infty} r^{-2}\left(V(r)-V_{0}(r)\right) \geq$ 0 . Combining this with positive mass theorem, if we further assume that the scalar curvature is non-negative, then the limit is zero if and only if $M$ is isometric to $\mathbb{R}^{3}$.

In [14], a notion of isoperimetric mass $m_{\mathrm{ISO}}(M)$ of an $\mathrm{AF}$ manifold is introduced by Huisken. It is defined as:

$$
m_{\mathrm{ISO}}=\limsup _{r \rightarrow \infty} \frac{2}{\mathcal{A}(r)}\left(V(r)-\frac{1}{6 \pi^{\frac{1}{2}}} \mathcal{A}^{\frac{3}{2}}(r)\right),
$$

where $V(r)$ is as before and $\mathcal{A}(r)$ is the area of the coordinate sphere with respect to the AF metric. Using the method of the proof of Theorem 1.2, Miao [17] proves that the isoperimetric mass and the ADM mass of an AF manifold are equal. We would like to thank Miao for allowing us to include his result in this work.

In the second part of the paper, we will consider the small-sphere limit of the Brown-York mass. Let $r$ be the distance to the fixed point $p$, and $R(p)$ is the scalar curvature evaluated at $p$. We have the following: 
Theorem 1.3. Let $(N, g)$ be a Riemannian manifold of dimension 3, $p$ be a fixed interior point on $N$ and $S_{r}$ be the geodesic sphere of radius $r$ center at $p$. For $r$ small enough, we have

$$
m_{\mathrm{BY}}\left(S_{r}\right)=\frac{r^{3}}{12} R(p)+\frac{r^{5}}{1440}\left[24|\operatorname{Ric}|^{2}(p)-13 R^{2}(p)+12 \Delta R(p)\right]+O\left(r^{6}\right),
$$

where $\Delta$ is Laplacian operator of $(M, g)$ and $|\mathrm{Ric}|$ is the norm of the Ricci curvature.

Let $M$ be an AF manifold with non-negative scalar curvature. Suppose the Brown-York mass of the coordinate spheres converge to zero, then $M$ must be the Euclidean space by Theorem 1.1 and the positive mass theorem in $[21,24]$. By Theorem 1.3, we have similar result near a point $p$. Namely, assume $R \geq 0$ in a neighborhood of $p$, then

$$
\lim _{r \rightarrow 0} \frac{m_{\mathrm{BY}}\left(S_{r}\right)}{r^{5}} \geq 0
$$

Equality holds if and only if $(N, g)$ is flat at $p$ and $R$ vanishes up to second order at $p$.

There are results on the small-sphere limits obtained by Brown-LauYork [5]. They consider a cut $S_{r}$ with an affine radius $r$ of the light cone at a point $p$ in a Lorentz manifold. Using the light cone of reference, they show that the expansion of the quasi-local energy is:

$$
E=\frac{4 \pi r^{3}}{3} T_{a b} n^{a} n^{b}+o\left(r^{3}\right)
$$

where $T_{a b}$ is the energy momentum tensor and $n$ is the unit future pointing time like vector defining the choice of the affine parameter. In our case, if we consider the Lorentz manifold $\mathbb{R} \times N$ with metric $\widetilde{g}=-d t^{2}+g$, and suppose the metric satisfies the Einstein equation:

$$
\widetilde{R}_{a b}-\frac{1}{2} \widetilde{R} \widetilde{g}_{a b}=8 \pi T_{a b}
$$

Let $n=\frac{\partial}{\partial t}$ be the future pointing unit normal, then

$$
\frac{R(p)}{12}=\frac{4 \pi r^{3}}{3} T_{a b} n^{a} n^{b}
$$


Hence, $r^{3}$ term of the expansion in our case is similar to that in [5]. However, we are using Euclidean reference and we only consider the time symmetric case.

In the case of vacuum space-time, Brown-Lau-York [5] also obtain the $r^{5}$ term in the expansion of $E$ as follows:

$$
E_{5}=\frac{r^{5}}{90} T_{a b c d} n^{a} n^{b} a^{c} n^{d}
$$

where $T_{a b c d}$ is the Bel-Robinson tensor, which depends only on the curvature tensor (and the metric). In Theorem 3.1, the space-time is not vacuum in general and is time symmetric. The coefficient of the term $r^{5}$ depends not only on the curvature tensor, but also on the derivative of the scalar curvature. For the sake of comparison, in our case, one can compute that $T_{0000}=\frac{1}{8}\left(4|\mathrm{Ric}|^{2}-R^{2}\right)$. We use the definition of Bel-Robinson tensor as in (5) of $[9]$.

Next we want to compare the expansion of the Hawking mass with the expansion of the Brown-York mass for small spheres. Recall the definition of the Hawking mass. Let $(\Omega, g)$ be a smooth 3 -manifold with boundary $\partial \Omega$ and let $H$ be the mean curvature on $\partial \Omega$ with respect to the outward unit normal, the Hawking quasi-local mass is defined as (see [11]):

\section{Definition 1.4.}

$$
m_{\mathrm{H}}(\partial \Omega)=\frac{|\partial \Omega|^{1 / 2}}{(16 \pi)^{3 / 2}}\left(16 \pi-\int_{\partial \Omega} H^{2} d \Sigma\right)
$$

where $d \Sigma$ is the volume element induced on $\partial \Omega$ by $g$ and $|\partial \Omega|$ is the area of $\partial \Omega$.

With the same notations and assumptions in Theorem 1.3, the expansion of $m_{\mathrm{H}}\left(S_{r}\right)$ is given by:

$$
m_{\mathrm{H}}\left(S_{r}\right)=\frac{r^{3}}{12} R(p)+\frac{r^{5}}{720}\left(6 \Delta R(p)-5 R^{2}(p)\right)+O\left(r^{6}\right)
$$

One can see that $m_{\mathrm{BY}}\left(S_{r}\right)$ and $m_{\mathrm{H}}\left(S_{r}\right)$ are equal up to the term with order $r^{3}$. However, the terms of order $r^{5}$ are different. In particular, if the scalar curvature is zero near $p$, but it is non-flat at $p$, then $r^{-5} m_{\mathrm{BY}}\left(S_{r}\right)>0$ and $m_{\mathrm{H}}\left(S_{r}\right)=O\left(r^{6}\right)$ for small $r$.

As in the large-sphere case, one can also compare $V(r)$ and $V_{0}(r)$, where $V(r)$ is the volume of the geodesic ball of radius $r$ at $p$ and $V_{0}(r)$ which is the volume of the region bounded by $S_{r}$ when embedded in $\mathbb{R}^{3}$. 
The paper is organized as follows. In Section 2, the limit of behavior of Brown-York mass in large spheres and volume comparison are proved; in Section 3, small-sphere limit of the Brown-York mass and the Hawking mass and small-sphere volume comparison are proved.

The authors would like to thank Robert Bartnik, Yanyan Li and Pengzi Miao for useful discussions.

\section{Large-sphere limit}

In this section, we will first prove the following theorem (Theorem 1.1).

Theorem 2.1. Let $(M, g)$ be an AF manifold of order $\tau>\frac{1}{2}$ with one end and let $S_{r}$ be the coordinate spheres in some admissible coordinates. Then

$$
\lim _{r \rightarrow \infty} m_{\mathrm{BY}}\left(S_{r}\right)=m_{\mathrm{ADM}}(M)
$$

Here $m_{\mathrm{BY}}\left(S_{r}\right)$ is the Brown-York quasi-local mass of $S_{r}$ and $m_{\mathrm{ADM}}(M)$ is the ADM mass of $M$.

Consider an AF manifold $(M, g)$ with coordinates $\left(x^{1}, x^{2}, x^{3}\right)$ so that $g_{i j}$ satisfies the decay conditions in Definition 1.3. Let $n=n^{i} \frac{\partial}{\partial x^{i}}$ be the unit outward normal of $S_{r}$ and $n_{i}=g_{i j} n^{j}$. Then

$$
n^{i}=\frac{g^{i j} x^{j}}{r|\nabla r|} \quad \text { and } \quad n_{i}=\frac{x^{i}}{r|\nabla r|}
$$

where $r=\left(\sum_{i=1}^{3}\left(x^{i}\right)^{2}\right)^{1 / 2}$. The metric induced on $S_{r}$ is $h_{i j}=g_{i j}-n_{i} n_{j}$ and the second fundamental form is $A_{i j}=h_{i}^{k} h_{j}^{l} n_{k ; l}$, where $n_{k ; l}$ is the covariant derivative of $n_{k}$ with respect to $g$.

Lemma 2.1. With the above notations and assumptions, on $S_{r}$ we have the following:

(i)

$$
A_{i j}=\frac{h_{i j}}{r}+O\left(r^{-1-\tau}\right), \quad H=\frac{2}{r}+O\left(r^{-1-\tau}\right), K=\frac{1}{r^{2}}+O\left(r^{-2-\tau}\right),
$$

where $H$ is the mean curvature and $K$ is the Gauss curvature of $S_{r}$. 
(ii)

$$
d \Sigma_{r}=\left(1+h^{i j} \sigma_{i j}+O\left(r^{-2 \tau}\right)\right)^{1 / 2} d \Sigma_{r}^{0}
$$

Hence,

$$
\mathcal{A}(r)=4 \pi r^{2}+\frac{1}{2} \int_{S_{r}} h^{i j} \sigma_{i j} d \Sigma_{r}+O\left(r^{2-2 \tau}\right)
$$

where $\mathcal{A}(r)$ is the area of $S_{r}$ with respect to $g$.

Proof. (i) is well-known, see [15]. For the sake of completeness, we derive it as follows:

$$
|\nabla r|^{2}=1-\frac{\sigma_{i j} x^{i} x^{j}}{r^{2}}+O\left(r^{-2 \tau}\right)
$$

and

$$
\begin{aligned}
\frac{\partial}{\partial x^{k}}\left(|\nabla r|^{2}\right) & =\frac{\partial}{\partial x^{k}}\left(g^{i j} \frac{x^{i} x^{j}}{r^{2}}\right) \\
& =\frac{\partial}{\partial x^{k}}\left[1+\left(g^{i j}-\delta_{i j}\right) \frac{x^{i} x^{j}}{r^{2}}\right] \\
& =O\left(r^{-1-\tau}\right) .
\end{aligned}
$$

So

$$
n^{i}=\frac{x^{i}}{r}+O\left(r^{-\tau}\right)
$$

and

$$
\begin{aligned}
n_{i ; j} & =\frac{\partial n_{i}}{\partial x^{j}}-\Gamma_{i j}^{k} n_{k} \\
& =\frac{\partial}{\partial x^{j}}\left(\frac{x^{i}}{r|\nabla r|}\right)+O\left(r^{-1-\tau}\right) \\
& =\left(\frac{\delta_{i j}}{r}-\frac{x^{i} x^{j}}{r^{3}}\right)+O\left(r^{-1-\tau}\right),
\end{aligned}
$$


where $\Gamma_{i j}^{k}$ are the Christoffel symbols. Let $h_{i}^{j}=g^{j k} h_{k i}$. Using the fact that $n$ has unit length, we have

$$
\begin{aligned}
A_{i j}-\frac{h_{i j}}{r} & =h_{i}^{k} h_{j}^{l} n_{k ; l}-\frac{h_{i j}}{r} \\
& =h_{j}^{l} n_{i ; l}-\frac{h_{i j}}{r} \\
& =n_{i ; j}-\left(\frac{\delta_{i j}}{r}-\frac{x^{i} x^{j}}{r^{3}}\right)+O\left(r^{-1-\tau}\right) \\
& =O\left(r^{-1-\tau}\right) .
\end{aligned}
$$

From this and the fact that the curvature of $M$ decays like $r^{-2-\tau}$, the estimates of $H$ and $K$ follow.

(ii) Let $e_{1}$ and $e_{2}$ be orthonormal frames on $S_{r}$ with respect to the Euclidean metric, then

$$
\begin{aligned}
d \Sigma_{r} & =\left(g\left(e_{1}, e_{1}\right) g\left(e_{2}, e_{2}\right)-g^{2}\left(e_{1}, e_{2}\right)\right)^{1 / 2} d \Sigma_{r}^{0} \\
& =\left(1+\sigma\left(e_{1}, e_{1}\right)+\sigma\left(e_{2}, e_{2}\right)+O\left(r^{-2 \tau}\right)\right)^{1 / 2} d \Sigma_{r}^{0} \\
& =\left[1+\left(e_{1}\left(x^{i}\right) e_{1}\left(x^{j}\right)+e_{2}\left(x^{i}\right) e_{2}\left(x^{j}\right)\right) \sigma_{i j}+O\left(r^{-2 \tau}\right)\right]^{1 / 2} d \Sigma_{r}^{0} \\
& =\left[1+\left(\nabla_{0} x^{i} \cdot \nabla_{0} x^{j}-\frac{\partial x^{i}}{\partial r} \frac{\partial x^{j}}{\partial r}\right) \sigma_{i j}+O\left(r^{-2 \tau}\right)\right]^{1 / 2} d \Sigma_{r}^{0} \\
& =\left[1+\left(\delta_{i j}-\frac{\partial x^{i}}{\partial r} \frac{\partial x^{j}}{\partial r}\right) \sigma_{i j}+O\left(r^{-2 \tau}\right)\right]^{1 / 2} d \Sigma_{r}^{0} \\
& =\left(1+h^{i j} \sigma_{i j}+O\left(r^{-2 \tau}\right)\right)^{1 / 2} d \Sigma_{r}^{0}
\end{aligned}
$$

where $\nabla_{0}$ is the derivative with respect to the Euclidean metric and '.' is the standard inner product in $\mathbb{R}^{3}$. The last statement follows from this immediately.

\section{Lemma 2.2.}

$$
\int_{S_{r}} H d \Sigma_{r}=\frac{\mathcal{A}(r)}{r}+4 \pi r-8 \pi m_{\mathrm{ADM}}(M)+o(1)
$$

as $r \rightarrow \infty$. 
Proof. Let $m=m_{\mathrm{ADM}}(M)$. By Lemma 2.1 and the first variational formula, we have

$$
\begin{aligned}
\frac{d}{d r} \mathcal{A}(r) & =\int_{S_{r}} \frac{1}{|\nabla r|} H d \Sigma_{r} \\
& =\int_{S_{r}} H d \Sigma_{r}+\int_{S_{r}} \frac{\sigma_{i j} x^{i} x^{j}}{r^{3}} d \Sigma_{r}+O\left(r^{1-2 \tau}\right)
\end{aligned}
$$

where we have used (2.2).

On the other hand, by Lemma 2.1, we have

$$
\begin{aligned}
\frac{d}{d r} \mathcal{A}(r)= & 8 \pi r+\frac{1}{2} \int_{S_{r}} \frac{\partial}{\partial r}\left(h^{i j} \sigma_{i j}\right) d \Sigma_{r}+\frac{1}{r} \int_{S_{r}} h^{i j} \sigma_{i j} d \Sigma_{r}+O\left(r^{1-2 \tau}\right) \\
= & 8 \pi r+\frac{1}{2} \int_{S_{r}} h^{i j} \sigma_{i j, k} \frac{x^{k}}{r} d \Sigma_{r}+\frac{1}{r} \int_{S_{r}} h^{i j} \sigma_{i j} d \Sigma_{r}+O\left(r^{1-2 \tau}\right) \\
= & 8 \pi r+\frac{1}{2} \int_{S_{r}} \frac{\sigma_{i i, k} x^{k}}{r} d \Sigma_{r}^{0}-\frac{1}{2} \int_{S_{r}} \frac{\sigma_{i j, k} x^{i} x^{j} x^{k}}{r^{3}} d \Sigma_{r}^{0} \\
& +\frac{1}{r} \int_{S_{r}} h^{i j} \sigma_{i j} d \Sigma_{r}+O\left(r^{1-2 \tau}\right),
\end{aligned}
$$

where $\sigma_{i j, k}=\frac{\partial \sigma_{i j}}{\partial x^{k}}$. Now, as in $[15,(5.17)]$ :

$$
\begin{aligned}
\int_{S_{r}} & \frac{\sigma_{i j, k} x^{i} x^{j} x^{k}}{r^{3}} d \Sigma_{r}^{0} \\
& =\int_{S_{r}} \frac{\partial}{\partial x^{k}}\left(\frac{\sigma_{i j} x^{j}}{r}\right) \frac{x^{i} x^{k}}{r^{2}} d \Sigma_{r}^{0} \\
& =-\int_{S_{r}}\left(\delta_{i k}-\frac{x^{i} x^{k}}{r^{2}}\right) \frac{\partial}{\partial x^{k}}\left(\frac{\sigma_{i j} x^{j}}{r}\right) d \Sigma_{r}^{0}+\int_{S_{r}} \frac{\partial}{\partial x^{i}}\left(\frac{\sigma_{i j} x^{j}}{r}\right) d \Sigma_{r}^{0} \\
& =-2 \int_{S_{r}} \frac{\sigma_{i j} x^{i} x^{j}}{r^{3}} d \Sigma_{r}^{0}+\int_{S_{r}} \frac{\sigma_{i j, i} x^{j}}{r} d \Sigma_{r}^{0}+\int_{S_{r}} \sigma_{i j}\left(\frac{\delta_{i j}}{r}-\frac{x^{i} x^{j}}{r^{3}}\right) d \Sigma_{r}^{0} \\
& =-2 \int_{S_{r}} \frac{\sigma_{i j} x^{i} x^{j}}{r^{3}} d \Sigma_{r}+\int_{S_{r}} \frac{\sigma_{i j, i} x^{j}}{r} d \Sigma_{r}^{0}+\frac{1}{r} \int_{S_{r}} h^{i j} \sigma_{i j} d \Sigma_{r}+O\left(r^{1-2 \tau}\right) .
\end{aligned}
$$


Combining this with (2.10), by Lemma 2.1 and the definition of ADM mass, we have

$$
\frac{d}{d r} \mathcal{A}(r)=\frac{\mathcal{A}(r)}{r}+4 \pi r+\int_{S_{r}} \frac{\sigma_{i j} x^{i} x^{j}}{r^{3}} d \Sigma_{r}^{0}-8 \pi m+o(1)
$$

By (2.9) and (2.12), the lemma follows.

By Lemma 2.1, if $r$ is large enough, then the Gauss curvature of $S_{r}$ is positive. So $S_{r}$ can be isometrically embedded in $\mathbb{R}^{3}$ uniquely up to an isometry of $\mathbb{R}^{3}$ by $[13,18-20]$. The following lemma says that the embedded surface (rescaled) is very close to the standard sphere as $r \rightarrow \infty$.

Lemma 2.3. Let $(M, g)$ be an AF 3-manifold with (1.1) and (1.2) for $\tau>\frac{1}{2}$, and let $S_{r}$ be coordinate spheres. For $r$ large enough, there is an isometrical embedding $X_{r}$ of $S_{r}$ in $\mathbb{R}^{3}$ such that:

$$
\begin{aligned}
X_{r} \cdot n_{0} & =r+O\left(r^{1-\tau}\right) \\
H_{0} & =\frac{2}{r}+H_{1} \text { with } H_{1}=O\left(r^{-1-\tau}\right)
\end{aligned}
$$

as $r \rightarrow+\infty$, where $n_{0}$ is the unit outward normal to the surface $X_{r}$, '.' is the inner product in $\mathbb{R}^{3}$ and $H_{0}$ is the mean curvature of $X_{r}$.

Proof. For $r>0$, define a map $x=r y$ and pull back the metric to the $y$ space. Let the pull back metric be $\hat{g}$. Let $\hat{h}$ be the induced metric on the coordinate spheres in $y$.

$$
\begin{aligned}
\hat{h}_{i j} & =\hat{g}_{i j}-\hat{n}_{i} \hat{n}_{j} \\
& =r^{2} g_{i j}-\hat{n}_{i} \hat{n}_{j}
\end{aligned}
$$

where $\hat{h}_{i j}=\hat{h}\left(\frac{\partial}{\partial y^{i}}, \frac{\partial}{\partial y^{j}}\right)$, etc. and $g_{i j}=g\left(\frac{\partial}{\partial x^{i}}, \frac{\partial}{\partial x^{j}}\right)$ etc. Also $\hat{n}_{i}=y^{i} /\left(\rho|\hat{\nabla} \rho|_{\hat{g}}\right)$ is the unit normal on $\left\{\rho=\left(\sum_{i=1}^{3}\left(y^{i}\right)^{2}\right)^{1 / 2}=\right.$ constant $\}$. Then

$$
|\hat{\nabla} \rho|_{\hat{g}}^{2}=r^{-2} g^{i j} \frac{y^{i} y^{j}}{\rho^{2}}
$$


Consider the following metric on $\Sigma_{\rho}=\{y|| y \mid=\rho\}$ :

$$
\begin{aligned}
d s_{r}^{2} & =r^{-2} \hat{h}_{i j} \\
& =g_{i j}-r^{-2} \hat{n}_{i} \hat{n}_{j} \\
& =g_{i j}-\frac{y^{i} y^{j}}{g^{k l} y^{k} y^{l}} .
\end{aligned}
$$

Clearly, the standard metric $h_{i j}^{0}$ on $\Sigma_{\rho}$ is

$$
d s_{0}^{2}=h_{i j}^{0}=\delta_{i j}-\frac{y^{i} y^{j}}{\rho^{2}} .
$$

Direct computations show

$$
\left\|d s_{r}^{2}-d s_{0}^{2}\right\|_{C^{3}\left(\Sigma_{\rho}\right)}=O\left(r^{-\tau}\right)
$$

for $\frac{1}{2} \leq \rho \leq 2$. Note that $\Sigma_{1}$ is the unit sphere. By [18, p. 353], we can find an isometric embedding $\hat{X}_{r}$ of $\left(\mathbb{S}^{2}, d s_{r}^{2}\right)$ into $\mathbb{R}^{3}$ such that

$$
\left\|\hat{X}_{r}-X_{0}\right\|_{C^{2}\left(\mathbb{S}^{2}\right)}=O\left(r^{-\tau}\right)
$$

where $X_{0}$ is the identity map. Since $X_{0} \cdot n_{0}=1$, where $n_{0}$ is the unit outward normal of the unit sphere, we have $\hat{X}_{r} \cdot n_{0, r}=1+O\left(r^{-\tau}\right)$, where $n_{0, r}$ is the unit outward normal of the surface $\hat{X}_{r}$. If we identify $S_{r}$ with metric induced by $g$ with $\left(\mathbb{S}^{2}, \hat{h}\right)$, then $X_{r}=r \hat{X}_{r}$ is an isometric embedding of $S_{r}$ with metric induced by $g$. From this it is easy to see that the first part of (2.13) is true.

By (2.18), we know that $\hat{H}_{0}-2=O\left(r^{-\tau}\right)$, where $\hat{H}_{0}$ is the mean curvature of $\hat{X}_{r}$. After rescaling $r \hat{X}_{r}$, we can get the second part of (2.13).

Lemma 2.4. Let $(M, g)$ be an AF manifold with the properties (1.1) and (1.2), and let $S_{r}$ be coordinate spheres. We have

$$
\int_{S_{r}} H_{0} d \Sigma_{r}=4 \pi r+\frac{\mathcal{A}(r)}{r}+O\left(r^{1-2 \tau}\right) .
$$

Proof. By Lemma 2.3, for $r$ large enough, we can find an isometric embed$\operatorname{ding} X_{r}$ of $S_{r}$ in $\mathbb{R}^{3}$ such that $X_{r} \cdot n_{0}=r+O\left(r^{1-\tau}\right)$. Let $H_{0}$ be the mean curvature with respect to the outward unit normal when $S_{r}$ is embedded 
in $\mathbb{R}^{3}$. By Lemma 2.1(i),

$$
\bar{K} \doteqdot K-\frac{1}{r^{2}}=O\left(r^{-2-\tau}\right) .
$$

By one of the Minkowski integral formulae [16, Lemma 6.2.9], we have

$$
\begin{aligned}
\int_{S_{r}} H_{0} d \Sigma_{r} & =2 \int_{S_{r}} K X_{r} \cdot n_{0} d \Sigma_{r} \\
& =2 \int_{S_{r}}\left(\frac{1}{r^{2}}+\bar{K}\right) X_{r} \cdot n_{0} d \Sigma_{r} \\
& =\frac{2}{r^{2}} \int_{S_{r}} X_{r} \cdot n_{0} d \Sigma_{r}+2 \int_{S_{r}} \bar{K} X_{r} \cdot n_{0} d \Sigma_{r} \\
& =\frac{6 V_{0}(r)}{r^{2}}+2 \int_{S_{r}} \bar{K}\left(r+O\left(r^{1-\tau}\right)\right) d \Sigma_{r} \\
& =\frac{6 V_{0}(r)}{r^{2}}+2 r \int_{S_{r}} \bar{K} d \Sigma_{r}+O\left(r^{1-2 \tau}\right) \\
& =\frac{6 V_{0}(r)}{r^{2}}+2 r \int_{S_{r}}\left(K-\frac{1}{r^{2}}\right) d \Sigma_{r}+O\left(r^{1-2 \tau}\right) \\
& =\frac{6 V_{0}(r)}{r^{2}}+8 \pi r-\frac{2 \mathcal{A}(r)}{r}+O\left(r^{1-2 \tau}\right)
\end{aligned}
$$

where $V_{0}(r)$ is the volume of the interior of the surface $X_{r}$ in $\mathbb{R}^{3}$. On the other hand, from Lemma 2.3, $H_{0}=\frac{2}{r}+H_{1}$ with $H_{1}=O\left(r^{-1-\tau}\right)$. By another Minkowski integral formula, we have

$$
\begin{aligned}
2 \mathcal{A}(r) & =\int_{S_{r}} H_{0} X \cdot n_{0} d \Sigma_{r} \\
& =\frac{6 V_{0}(r)}{r}+\int_{S_{r}} H_{1} X \cdot n_{0} d \Sigma_{r} \\
& =\frac{6 V_{0}(r)}{r}+r \int_{S_{r}} H_{1} d \Sigma_{r}+O\left(r^{2-2 \tau}\right) \\
& =\frac{6 V_{0}(r)}{r}-2 \mathcal{A}(r)+r \int_{S_{r}} H_{0} d \Sigma_{r}+O\left(r^{2-2 \tau}\right) .
\end{aligned}
$$

So

$$
\int_{S_{r}} H_{0} d \Sigma_{r}=-\frac{6 V_{0}(r)}{r^{2}}+\frac{4 \mathcal{A}(r)}{r}+O\left(r^{1-2 \tau}\right) .
$$

From (2.20) and (2.22), the lemma follows. 
Proof of Theorem 2.1. The theorem follows immediately from Lemmas 2.2 and 2.4.

In Theorem 2.1, $S_{r}$ can be replaced by slightly deformed spheres. More precisely, we have:

Corollary 2.1. Same assumptions as in Theorem 2.1. Suppose $\rho$ is a smooth function on $M$ such that

$$
|\rho-r|+r|\partial(\rho-r)|+r^{2}|\partial \partial(\rho-r)|+r^{3}|\partial \partial \partial(\rho-r)|=O\left(r^{\kappa}\right)
$$

for some $0<\kappa<1-\tau$. Then

$$
\lim _{\rho \rightarrow \infty} m_{\mathrm{BY}}\left(\Sigma_{\rho}\right)=m_{\mathrm{ADM}}(M)
$$

where $\Sigma_{\rho}$ is the level set of the smooth function $\rho$.

Proof. Let $y=\frac{\rho}{r} x=F(x)$. Then one can show that $y$ is also a coordinate system of $M$ at infinity so that the metric tensor in this coordinates satisfies the decay conditions (1.1) and (1.2). Note that $\Sigma_{\rho}$ is nothing but the coordinate spheres in the $y$-coordinates. Hence, the corollary follows from the uniqueness of ADM mass by [2].

With the notations as in the proof of Theorem 2.1. Let $V(r)$ be the volume with respect to an AF metric $g$ of the region inside $S_{r}$. We can compare $V(r)$ and $V_{0}(r)$ (Theorem 1.2):

Theorem 2.2. With the above notations. Let $(M, g)$ be an AF manifold of order $\tau>\frac{1}{2}$ with one end. Then

$$
V_{0}(r)-V(r)=-2 m_{\mathrm{ADM}} \pi r^{2}+o\left(r^{2}\right)
$$

Proof. Let $m=m_{A D M}$. With the same notations as in the proof of Theorem 2.1 , by (2.2) and the co-area formula we have

$$
\begin{aligned}
V^{\prime}(r) & =\int_{S_{r}} \frac{1}{|\nabla r|} d \Sigma_{r} \\
& =\mathcal{A}(r)+\frac{1}{2} \int_{S_{r}} \frac{\sigma_{i j} x^{i} x^{j}}{r^{2}} d \Sigma_{r}+O\left(r^{2-2 \tau}\right) .
\end{aligned}
$$


Here and below is the derivative with respect to $r$. On the other hand, by (2.8) and (2.9) we have

$$
\mathcal{A}^{\prime}(r)=\frac{\mathcal{A}(r)}{r}+4 \pi r-8 \pi m+\int_{S_{r}} \frac{\sigma_{i j} x^{i} x^{j}}{r^{3}} d \Sigma_{r}+o(1) .
$$

Eliminating the term $\int_{S_{r}} \frac{\sigma_{i j} x^{i} x^{j}}{r^{3}} d \Sigma_{r}$ from (2.25) and (2.26), we have

$$
\mathcal{A}^{\prime}(r)=\frac{\mathcal{A}(r)}{r}+4 \pi r-8 \pi m+\frac{1}{r}\left(2 V^{\prime}(r)-2 \mathcal{A}(r)\right)+o(1)
$$

Hence,

$$
(r \mathcal{A}(r))^{\prime}=4 \pi r^{2}-8 \pi m r+2 V^{\prime}(r)+o(r)
$$

and

$$
V(r)=\frac{1}{2} r \mathcal{A}(r)-\frac{2 \pi r^{3}}{3}+2 \pi m r^{2}+o\left(r^{2}\right)
$$

On the other hand, by (2.20) and (2.21), we have

$$
V_{0}(r)=\frac{1}{2} r \mathcal{A}(r)-\frac{2 \pi r^{3}}{3}+O\left(r^{3-2 \tau}\right) .
$$

Hence

$$
V_{0}(r)-V(r)=-2 \pi m r^{2}+o\left(r^{2}\right)
$$

because $\tau>\frac{1}{2}$.

Combine this with positive mass theorem, we have the following:

Corollary 2.2. With above notations, let $(M, g)$ be an AF manifold of order $\tau>\frac{1}{2}$. If the scalar curvature is non-negative, then

$$
\lim _{r \rightarrow+\infty} \frac{V(r)-V_{0}(r)}{r^{2}} \geq 0
$$

and equality holds if and only if $(M, g)$ is isometric to $\mathbb{R}^{3}$.

From the proof of Theorem 2.2, Miao [17] is able to obtain the following result. Thanks to Pengzi Miao, we include the result and the proof here.

Corollary 2.3. In an AF manifold $M$, the ADM mass and the isoperimetric mass introduced by Huisken [14] are equal. 
Proof. Recall that the isoperimetric mass of $M$ is defined as

$$
m_{\mathrm{ISO}}=\limsup _{r \rightarrow \infty} \frac{2}{\mathcal{A}(r)}\left(V(r)-\frac{1}{6 \pi^{1 / 2}} \mathcal{A}^{3 / 2}(r)\right) .
$$

Now by $(2.28)$

(2.30)

$$
\begin{aligned}
\frac{2}{\mathcal{A}(r)} & \left(V(r)-\frac{1}{6 \pi^{1 / 2}} \mathcal{A}^{3 / 2}(r)\right) \\
& =r+\frac{1}{\mathcal{A}(r)}\left(4 \pi m r^{2}-\frac{4 \pi r^{3}}{3}\right)-\frac{1}{3 \pi^{1 / 2}} \mathcal{A}^{1 / 2}(r)+o(1) \\
& =r+\left(m-\frac{r}{3}\right)\left(1-\mathcal{I}+O\left(r^{-2 \tau}\right)\right)-\frac{2 r}{3}\left(1+\frac{1}{2} \mathcal{I}+O\left(r^{-2 \tau}\right)\right)+o(1) \\
& =m+o(1)
\end{aligned}
$$

where

$$
\mathcal{I}=\frac{1}{8 \pi r^{2}} \int_{S_{r}} h^{i j} \sigma_{i j} d \Sigma_{r}=O\left(r^{-\tau}\right)
$$

so that

$$
\mathcal{A}(r)=4 \pi r^{2}\left(1+\mathcal{I}+O\left(r^{-2 \tau}\right)\right)
$$

see Lemma 2.1(ii). From this the result follows.

\section{Small-sphere limit}

In this section, we will first study the small-sphere limit of the Brown-York mass of geodesic spheres up to order $r^{5}$, where $r$ is the geodesic distance from a fixed point. Let $\left(N^{3}, g\right)$ be a three-dimensional manifold and let $p \in N$. Let $\left\{x^{i}\right\}$ be the normal coordinates near $p$. By [22, Chapter 5], we have the following expansion of $g$ near $p$ :

Lemma 3.1. For any point $x$ close to $p$, the metric components of $g$ in the normal coordinates can be expressed as

$$
\begin{aligned}
g_{i j}(x)= & \delta_{i j}+\frac{1}{3} R_{i k l j}(p) x^{k} x^{l}+\frac{1}{6} R_{i k l j ; m}(p) x^{k} x^{l} x^{m} \\
& +\left(\frac{1}{20} R_{i k l j ; m n}(p)+\frac{2}{45} R_{i k l s}(p) R_{j m n s}(p)\right) x^{k} x^{l} x^{m} x^{n}+O\left(r^{5}\right)
\end{aligned}
$$


and

$$
\begin{aligned}
g= & \operatorname{det}\left(g_{i j}\right) \\
= & 1-\frac{1}{3} R_{i j}(p) x^{i} x^{j}-\frac{1}{6} R_{i j ; k}(p) x^{i} x^{j} x^{k}-\left(\frac{1}{20} R_{i j ; k l}(p)\right. \\
& \left.+\frac{1}{90} R_{h i j m}(p) R_{h k l m}(p)-\frac{1}{18} R_{i j}(p) R_{k l}(p)\right) x^{i} x^{j} x^{k} x^{l}+O\left(r^{5}\right),
\end{aligned}
$$

where $r$ is the geodesic distance from $p, R_{i j k l}$ is the Riemannian curvature tensor, $R_{i j}$ is the Ricci curvature and $R$ is the scalar curvature with respect to the metric $g$, and $R_{i k l j ; m}$ is the covariant derivative of $R_{i j k l}$ etc.

In our notations, the sectional curvature is non-negative if $R_{i j i j} \geq 0$. In the following, we always assume that the normal coordinates are chosen so that at $p$ the Ricci curvature is of the form $R_{i j}=\lambda_{i} \delta_{i j}$ where $\lambda_{1}, \lambda_{2}, \lambda_{3}$ are the eigenvalues of $R_{i j}$.

Lemma 3.2. Let $\mathcal{A}(r)$ be the area of geodesic sphere $S_{r}=\{|x|=r\}$ with radius $r$ in $(N, g)$ with center at $p$, then:

$$
\mathcal{A}(r)=4 \pi r^{2}+A_{4}+A_{6}+O\left(r^{7}\right)
$$

where

$$
A_{4}=-\frac{2 \pi r^{4}}{9} R, \quad A_{6}=\frac{\pi r^{6}}{675}\left(4 R^{2}-2|\mathrm{Ric}|^{2}-9 \Delta R\right)
$$

where $\Delta$ is the Laplacian operator with respect to metric $g$ and $|\mathrm{Ric}|$ is the norm of the Ricci tensor. Here all the terms involving curvature are evaluated at $p$.

Proof. By (3.2)

$$
\sqrt{g}=1+\frac{1}{2}\left(b_{2}+b_{3}+b_{4}\right)-\frac{1}{8} b_{2}^{2}+O\left(r^{5}\right),
$$

where

$$
\begin{aligned}
b_{2} & =-\frac{1}{3} R_{i j} x^{i} x^{j} \\
b_{3} & =-\frac{1}{6} R_{i j, k} x^{i} x^{j} x^{k} \\
b_{4} & =-\left(\frac{1}{20} R_{i j, k l}+\frac{1}{90} R_{h i j m} R_{h k l m}-\frac{1}{18} R_{i j} R_{k l}\right) x^{i} x^{j} x^{k} x^{l} .
\end{aligned}
$$


Hence

$$
V(r)=\int_{B_{r}} \sqrt{g} d v_{0},
$$

where $d v_{0}$ is the volume element with respect to Euclidean metric and $B_{r}=$ $\{x|| x \mid<r\}$. Since $\left|\frac{\partial}{\partial r}\right|=1$ in $g$ metric,

$$
\begin{aligned}
\mathcal{A}(r) & =V^{\prime}(r) \\
& =4 \pi r^{2}+\int_{S_{r}}\left[\frac{1}{2}\left(b_{2}+b_{3}+b_{4}\right)-\frac{1}{8} b_{2}^{2}+O\left(r^{5}\right)\right] d \Sigma_{r}^{0} \\
& =4 \pi r^{2}+\frac{1}{2} \int_{S_{r}} b_{2} d \Sigma_{r}^{0}+\frac{1}{2} \int_{S_{r}}\left(b_{4}-\frac{1}{4} b_{2}^{2}\right) d \Sigma_{r}^{0}+O\left(r^{7}\right)
\end{aligned}
$$

where $d \Sigma_{r}^{0}$ is the area element of $S_{r}$ with respect to the Euclidean metric. Since $\int_{S_{r}}\left(x^{i}\right)^{2} d \Sigma_{r}^{0}=\frac{4}{3} \pi r^{4}$, by (3.5) and the fact that $R_{i j} x^{i} x^{j}=\sum_{i=1}^{3} \lambda_{i}\left(x^{i}\right)^{2}$,

$$
\frac{1}{2} \int_{S_{r}} b_{2} d \Sigma_{r}^{0}=-\frac{2 \pi r^{4}}{9} R
$$

Noting that

$$
\begin{aligned}
b_{4} & =-\frac{1}{20} R_{i j ; k l} x^{i} x^{j} x^{k} x^{l}-\frac{1}{90} \sum_{h, m}\left(\sum_{i j} R_{h i j m} x^{i} x^{j}\right)^{2}+\frac{1}{18}\left(\sum_{i j} R_{i j} x^{i} x^{j}\right)^{2} \\
& =-\frac{1}{20} R_{i j ; k l} x^{i} x^{j} x^{k} x^{l}-\frac{1}{90} \sum_{i, j}\left(\sum_{k, l} R_{i k l j} x^{k} x^{l}\right)^{2}+\frac{1}{2} b_{2}^{2} .
\end{aligned}
$$

Let us first compute $\int_{S_{r}}\left(R_{i j} x^{i} x^{j}\right)^{2} d \Sigma_{r}^{0}$. By symmetry,

$$
\int_{S_{r}}\left(x^{i}\right)^{4} d \Sigma_{r}^{0}=\frac{4}{5} \pi r^{6}, \text { for } i=1,2,3,
$$

and for $i \neq j$,

$$
\int_{S_{r}}\left(x^{i} x^{j}\right)^{2} d \Sigma_{r}^{0}=\frac{4}{15} \pi r^{6}
$$


We have

$$
\begin{aligned}
\int_{S_{r}}\left(R_{i j} x^{i} x^{j}\right)^{2} d \Sigma_{r}^{0} & =\int_{S_{r}}\left(\sum_{i} \lambda_{i}\left(x^{i}\right)^{2}\right)^{2} d \Sigma_{r}^{0} \\
& =\frac{4}{5} \pi r^{6} \sum_{i} \lambda_{i}^{2}+\frac{4}{15} \pi r^{6} \sum_{i \neq j} \lambda_{i} \lambda_{j} \\
& =\frac{4}{15} \pi r^{6}\left(R^{2}+2|\mathrm{Ric}|^{2}\right)
\end{aligned}
$$

Since the $\operatorname{dim} N=3$, by [10, p. 276], at $p$

$$
\begin{aligned}
R_{i j k l} & =g_{i k} R_{j l}-g_{i l} R_{j k}-g_{j k} R_{i l}+g_{j l} R_{i k}-\frac{1}{2} R\left(g_{i k} g_{j l}-g_{i l} g_{j k}\right) \\
& =\delta_{i k} R_{j l}-\delta_{i l} R_{j k}-\delta_{j k} R_{i l}+\delta_{j l} R_{i k}-\frac{1}{2} R\left(\delta_{i k} \delta_{j l}-\delta_{i l} \delta_{j k}\right),
\end{aligned}
$$

and hence on $S_{r}$ :

$$
R_{i j k l} x^{j} x^{k}=\left(\lambda_{i}+\lambda_{l}-\frac{R}{2}\right) x^{i} x^{l}-\delta_{i l}\left(\sum_{k} \lambda_{k}\left(x^{k}\right)^{2}+\left(\lambda_{i}-\frac{R}{2}\right) r^{2}\right) .
$$

Using (3.10), we have

$$
\begin{aligned}
\int_{S_{r}} \sum_{i \neq l}\left(\sum_{j, k} R_{i j k l} x^{j} x^{k}\right)^{2} d \Sigma_{r}^{0} & =\int_{S_{r}} \sum_{i \neq l}\left(\lambda_{l}+\lambda_{i}-\frac{R}{2}\right)^{2}\left(x^{i} x^{l}\right)^{2} d \Sigma_{r}^{0} \\
& =\frac{4}{15} \pi r^{6} \sum_{i \neq l}\left(\lambda_{l}+\lambda_{i}-\frac{R}{2}\right)^{2} \\
& =\frac{4}{15} \pi r^{6}\left(2|\mathrm{Ric}|^{2}-\frac{R^{2}}{2}\right) .
\end{aligned}
$$


Clearly, by (3.13), (3.9) and (3.10), we have

$$
\begin{aligned}
& \int_{S_{r}}\left(\sum_{j k} R_{1 j k 1} x^{j} x^{k}\right)^{2} d \Sigma_{r}^{0} \\
& =\int_{S_{r}}\left(\left(2 \lambda_{1}-\frac{R}{2}\right)\left(x^{1}\right)^{2}-\sum_{k} \lambda_{k}\left(x^{k}\right)^{2}-\left(\lambda_{1}-\frac{R}{2}\right) r^{2}\right)^{2} d \Sigma_{r}^{0} \\
& =\pi r^{6}\left(-\frac{4}{15} \lambda_{1}^{2}+\frac{8}{15} R \lambda_{1}-\frac{4}{15} R^{2}+\frac{8}{15}|\mathrm{Ric}|^{2}\right)
\end{aligned}
$$

We have similar formula for the case $i=l=2$ or 3 . So

$$
\int_{S_{r}} \sum_{i=l}\left(\sum_{j, k} R_{i j k l} x^{j} x^{k}\right)^{2} d \Sigma_{r}^{0}=\pi r^{6}\left(\frac{20}{15}|\mathrm{Ric}|^{2}-\frac{4}{15} R^{2}\right) .
$$

By (3.14) and (3.16) we have

$$
\int_{S_{r}} \sum_{i, l}\left(\sum_{j, k} R_{i j k l} x^{j} x^{k}\right)^{2} d \Sigma_{r}^{0}=\frac{1}{15}\left(28|\mathrm{Ric}|^{2}-6 R^{2}\right) \pi r^{6}
$$

Finally, let us compute $\int_{S_{r}} \sum_{i, j, k, l} R_{i j ; k l} x^{i} x^{j} x^{k} x^{l} d \Sigma_{r}^{0}$. By symmetry $\int_{S_{r}} R_{i j ; k l} x^{i} x^{j} x^{k} x^{l} d \Sigma_{r}^{0}=0$ unless $x^{i} x^{j} x^{k} x^{l}$ is of the form $\left(x^{m}\right)^{4}$, or $\left(x^{m}\right)^{2}\left(x^{n}\right)^{2}$ with $m \neq n$.

$$
\begin{aligned}
\sum_{j, k, l} & \int_{S_{r}} R_{1 j ; k l} x^{1} x^{j} x^{k} x^{l} d \Sigma_{r}^{0} \\
= & \int_{S_{r}} R_{11 ; 11}\left(x^{1}\right)^{4} d \Sigma_{r}^{0}+\int_{S_{r}} R_{11 ; 22}\left(x^{1} x^{2}\right)^{2} d \Sigma_{r}^{0}+\int_{S_{r}} R_{11 ; 33}\left(x^{1} x^{3}\right)^{2} d \Sigma_{r}^{0} \\
& +\int_{S_{r}}\left(R_{12 ; 12}+R_{12 ; 21}\right)\left(x^{1} x^{2}\right)^{2} d \Sigma_{r}^{0}+\int_{S_{r}}\left(R_{13 ; 13}+R_{13 ; 31}\right)\left(x^{1} x^{3}\right)^{2} d \Sigma_{r}^{0} \\
= & \frac{4}{5} \pi r^{6} R_{11 ; 11}+\frac{4}{15} \pi r^{6}\left(R_{12 ; 12}+R_{12 ; 21}+R_{13 ; 13}+R_{13 ; 31}+R_{11 ; 22}+R_{11 ; 33}\right) .
\end{aligned}
$$

Similarly, one can prove that

$$
\begin{aligned}
\sum_{j, k, l} \int_{S_{r}} R_{2 j, k l} x^{2} x^{j} x^{k} x^{l} d \Sigma_{r}^{0}= & \frac{4}{5} \pi r^{6} R_{22 ; 22}+\frac{4}{15} \pi r^{6}\left(R_{21 ; 21}+R_{21 ; 12}\right. \\
& \left.+R_{23 ; 23}+R_{23 ; 32}+R_{22 ; 11}+R_{22 ; 33}\right),
\end{aligned}
$$


and

$$
\begin{aligned}
\sum_{j, k, l} \int_{S_{r}} R_{3 j, k l} x^{3} x^{j} x^{k} x^{l} d \Sigma_{r}^{0}= & \frac{4}{5} \pi r^{6} R_{33 ; 33}+\frac{4}{15} \pi r^{6}\left(R_{31 ; 31}+R_{31 ; 13}\right. \\
& \left.+R_{32 ; 32}+R_{32 ; 23}+R_{33 ; 11}+R_{33 ; 22}\right) .
\end{aligned}
$$

Hence,

$$
\sum_{i, j, k, l} \int_{S_{r}} R_{i j ; k l} x^{i} x^{j} x^{k} x^{l} d \Sigma_{r}^{0}=\frac{4}{15} \pi r^{6} \sum_{i, j}\left(R_{i i ; j j}+2 R_{i j ; i j}\right)
$$

By the second Bianchi identity, we see that

$$
\sum_{i} R_{i j ; i j}=\frac{1}{2} R_{; j j}
$$

Therefore, we have

$$
\sum_{i, j, k, l} \int_{S_{r}} R_{i j, k l} x^{i} x^{j} x^{k} x^{l} d \Sigma_{r}^{0}=\frac{8 \pi r^{6}}{15} \Delta R(p)
$$

The lemma follows from (3.5), (3.6), (3.7), (3.8), (3.11), (3.15), (3.17) and $(3.22)$.

Corollary 3.1. With the notations and assumptions as in Lemma 3.2, let $H$ be the mean curvature of $S_{r}$ with respect to $g$, then

$$
\int_{S_{r}} H d \Sigma_{r}=8 \pi r+\frac{4 A_{4}+6 A_{6}}{r}+O\left(r^{6}\right) .
$$

Proof. By the fact that $|\nabla r|=1$, we have

$$
\int_{S_{r}} H d \Sigma_{r}=\frac{d}{d r} \mathcal{A}(r)
$$

The corollary then follows from Lemma 3.2.

By [18], and the fact that for $r$ small $\left(S_{r},\left.g\right|_{S_{r}}\right)$ has positive Gauss curvature, one can isometrically embed $\left(S_{r},\left.g\right|_{S_{r}}\right)$ in $\mathbb{R}^{3}$. 
Lemma 3.3. For $r$ small enough, there is an isometric embedding $Z$ of geodesic sphere $S_{r}$ into $\mathbb{R}^{3}$ such that

$$
Z \cdot n=r+\frac{r^{3}}{6}\left(\frac{R}{2}-2 R_{i j} \frac{x^{i} x^{j}}{r^{2}}\right)+O\left(r^{4}\right),
$$

where $n$ is the outward unit normal vector of $Z\left(S_{r}\right)$ in $\mathbb{R}^{3}$ and '.' is the inner product in $\mathbb{R}^{3}$.

Proof. For $r>0$, we define a map $x=r y$ and pull back the metric $g$ to the $y$ space and let $h$ be the metric $r^{-2} g$ induced on the unit sphere $\mathbb{S}^{2}$ in the $y$ space. As in the proof of Lemma 2.3, in order to prove the lemma, it is sufficient to prove that for $r$ small, we can find an isometric embedding $Z_{r}$ of $\left(\mathbb{S}^{2}, h\right)$ in $\mathbb{R}^{3}$ such that

$$
Z_{r} \cdot n_{r}=1+\frac{r^{2}}{6}\left(\frac{R}{2}-2 \sum \lambda_{k}\left(y^{k}\right)^{2}\right)+O\left(r^{3}\right)
$$

where $n_{r}$ is the unit outward normal of $Z_{r}\left(\mathbb{S}^{2}\right)$.

Let $\tilde{h}$ be the induced metric of $r^{-2} \widetilde{g}$ on the unit sphere $\mathbb{S}^{2}$, where

$$
\widetilde{g}_{i j}=\widetilde{g}\left(\frac{\partial}{\partial y^{i}}, \frac{\partial}{\partial y^{j}}\right)=r^{2}\left(\delta_{i j}+\frac{r^{2}}{3} R_{i k l j} y^{k} y^{l}\right) \text {. }
$$

Let $\hat{h}$ be the metric on $\mathbb{S}^{2}$ induced by the pull back of the Euclidean metric given by the embedding $\hat{Z}=\left(z^{1}, z^{2}, z^{3}\right)$ in $\mathbb{R}^{3}$ where

$$
\begin{aligned}
& z^{1}=y^{1}\left(1+\frac{r^{2}}{6}\left(\frac{R}{2}-\lambda_{1}-\sum_{i} \lambda_{i}\left(y^{i}\right)^{2}\right)\right) \\
& z^{2}=y^{2}\left(1+\frac{r^{2}}{6}\left(\frac{R}{2}-\lambda_{2}-\sum_{i} \lambda_{i}\left(y^{i}\right)^{2}\right)\right) \\
& z^{3}=y^{3}\left(1+\frac{r^{2}}{6}\left(\frac{R}{2}-\lambda_{3}-\sum_{i} \lambda_{i}\left(y^{i}\right)^{2}\right)\right) .
\end{aligned}
$$

We claim that

$$
\|h-\tilde{h}\|_{C^{3}}+\|\hat{h}-\tilde{h}\|_{C^{3}}=O\left(r^{3}\right),
$$

where the norm is computed with respect to the standard metric. Suppose the claim is true, then by [18], we can conclude that there are isometric 
embeddings $Z_{r}, \tilde{Z}$ and $\hat{Z}$ for $\left(\mathbb{S}^{2}, h\right),\left(\mathbb{S}^{2}, \tilde{h}\right)$ and $\left(\mathbb{S}^{2}, \hat{h}\right)$, respectively, such that

$$
\left\|Z_{r} \cdot n_{r}-\hat{Z} \cdot \hat{n}\right\|_{C^{0}\left(\mathbb{S}^{2}\right)}=O\left(r^{3}\right)
$$

where $\hat{n}$ is the unit outward normal of $\hat{Z}\left(\mathbb{S}^{2}\right)$. Then we can prove the lemma by computing $\hat{Z} \cdot \hat{n}$.

Let us first prove the claim and then compute $\hat{Z} \cdot \hat{n}$. It is easy to see that $\|h-\tilde{h}\|_{C^{3}}=O\left(r^{3}\right)$ by the expression of $g$ in Lemma 3.1 and the definition of $\widetilde{g}$.

To find $\tilde{h}$, let $r^{-2} \widetilde{g}_{i j}=\delta_{i j}+\widetilde{\sigma}_{i j}$, and let $\lambda_{i j}=-\left(\lambda_{i}+\lambda_{j}\right)+\frac{R}{2}$. By $(3.13)$, we have

$$
\begin{aligned}
& \widetilde{\sigma}_{11}=\frac{r^{2}}{3}\left(\lambda_{12}\left(y^{2}\right)^{2}+\lambda_{13}\left(y^{3}\right)^{2}\right), \\
& \widetilde{\sigma}_{22}=\frac{r^{2}}{3}\left(\lambda_{12}\left(y^{1}\right)^{2}+\lambda_{23}\left(y^{3}\right)^{2}\right), \\
& \widetilde{\sigma}_{33}=\frac{r^{2}}{3}\left(\lambda_{13}\left(y^{1}\right)^{2}+\lambda_{23}\left(y^{2}\right)^{2}\right), \\
& \widetilde{\sigma}_{i j}=-\frac{r^{2}}{3} \lambda_{i j} y^{i} y^{j}, i \neq j .
\end{aligned}
$$

In the above last equation, the repeated indices is not taken summation.

Let $e_{1}=\partial_{\theta}=a_{i} \partial_{i}, e_{2}=(\sin \theta)^{-1} \partial_{\phi}=b_{i} \partial_{i}$. Then

$$
\begin{aligned}
& a_{1}=\cos \theta \cos \phi, \quad a_{2}=\cos \theta \sin \phi, \quad a_{3}=-\sin \theta \\
& b_{1}=-\sin \phi, \quad b_{2}=\cos \phi, \quad b_{3}=0 .
\end{aligned}
$$

Note that

$$
y^{1}=\sin \theta \cos \phi, \quad y^{2}=\sin \theta \sin \phi, \quad y^{3}=\cos \theta .
$$

Hence, in the basis $\left\{e_{1}, e_{2}\right\}, \tilde{h}$ is given by

$$
\begin{aligned}
& \tilde{h}_{11}=1+\frac{r^{2}}{3}\left(\lambda_{23} \sin ^{2} \phi+\lambda_{31} \cos ^{2} \phi\right), \\
& \tilde{h}_{12}=\frac{r^{2}}{3}\left(-\lambda_{13}+\lambda_{23}\right) \cos \theta \cos \phi \sin \phi, \\
& \tilde{h}_{22}=1+\frac{r^{2}}{3}\left(\lambda_{12} \sin ^{2} \theta+\lambda_{13} \cos ^{2} \theta \sin ^{2} \phi+\lambda_{23} \cos ^{2} \theta \cos ^{2} \phi\right) .
\end{aligned}
$$


Next we want to compute $\hat{h}$.

$$
\begin{aligned}
& \left(z^{1}\right)_{\theta}=\left(y^{1}\right)_{\theta}\left(1+\frac{r^{2}}{6}\left(\frac{R}{2}-\lambda_{1}-\sum_{i} \lambda_{i}\left(y^{i}\right)^{2}\right)\right)-\frac{r^{2}}{6} y^{1}\left(\sum_{i} \lambda_{i}\left(y^{i}\right)^{2}\right)_{\theta} \\
& \left(z^{2}\right)_{\theta}=\left(y^{2}\right)_{\theta}\left(1+\frac{r^{2}}{6}\left(\frac{R}{2}-\lambda_{2}-\sum_{i} \lambda_{i}\left(y^{i}\right)^{2}\right)\right)-\frac{r^{2}}{6} y^{2}\left(\sum_{i} \lambda_{i}\left(y^{i}\right)^{2}\right)_{\theta} \\
& \left(z^{3}\right)_{\theta}=\left(y^{3}\right)_{\theta}\left(1+\frac{r^{2}}{6}\left(\frac{R}{2}-\lambda_{3}-\sum_{i} \lambda_{i}\left(y^{i}\right)^{2}\right)\right)-\frac{r^{2}}{6} y^{3}\left(\sum_{i} \lambda_{i}\left(y^{i}\right)^{2}\right)_{\theta},
\end{aligned}
$$

and

$$
\begin{aligned}
& \left(z^{1}\right)_{\phi}=\left(y^{1}\right)_{\phi}\left(1+\frac{r^{2}}{6}\left(\frac{R}{2}-\lambda_{1}-\sum_{i} \lambda_{i}\left(y^{i}\right)^{2}\right)\right)-\frac{r^{2}}{6} y^{1}\left(\sum_{i} \lambda_{i}\left(y^{i}\right)^{2}\right)_{\phi} \\
& \left(z^{2}\right)_{\phi}=\left(y^{2}\right)_{\phi}\left(1+\frac{r^{2}}{6}\left(\frac{R}{2}-\lambda_{2}-\sum_{i} \lambda_{i}\left(y^{i}\right)^{2}\right)\right)-\frac{r^{2}}{6} y^{2}\left(\sum_{i} \lambda_{i}\left(y^{i}\right)^{2}\right)_{\phi} \\
& \left(z^{3}\right)_{\phi}=\left(y^{3}\right)_{\phi}\left(1+\frac{r^{2}}{6}\left(\frac{R}{2}-\lambda_{3}-\sum_{i} \lambda_{i}\left(y^{i}\right)^{2}\right)\right)-\frac{r^{2}}{6} y^{3}\left(\sum_{i} \lambda_{i}\left(y^{i}\right)^{2}\right)_{\phi} .
\end{aligned}
$$

Hence,

$$
\begin{aligned}
\hat{Z}_{\theta} \cdot \hat{Z}_{\theta}= & \left(z^{1}\right)_{\theta}^{2}+\left(z^{2}\right)_{\theta}^{2}+\left(z^{3}\right)_{\theta}^{2} \\
= & \sum_{i}\left(y^{i}\right)_{\theta}^{2}\left(1+\frac{r^{2}}{3}\left(\frac{R}{2}-\lambda_{i}-\sum_{k} \lambda_{k}\left(y^{k}\right)^{2}\right)\right) \\
& -\frac{r^{2}}{3} \sum_{i} y^{i}\left(y^{i}\right)_{\theta}\left(\sum_{i} \lambda_{i}\left(y^{i}\right)^{2}\right)_{\theta}+O\left(r^{4}\right) \\
= & \left.1+\frac{r^{2}}{3}\left[\frac{R}{2}-\sum_{i} \lambda_{i}\left(\left(y^{i}\right)_{\theta}^{2}+\left(y^{i}\right)^{2}\right)\right)\right]+O\left(r^{4}\right) \\
= & 1+\frac{r^{2}}{3}\left(\frac{R}{2}-\lambda_{1} \cos ^{2} \phi-\lambda_{2} \sin ^{2} \phi-\lambda_{3}\right)+O\left(r^{4}\right) \\
= & 1+\frac{r^{2}}{3}\left(\lambda_{13} \cos ^{2} \phi+\lambda_{23} \sin ^{2} \phi\right)+O\left(r^{4}\right)
\end{aligned}
$$




$$
\begin{aligned}
\hat{Z}_{\phi} \cdot \hat{Z}_{\phi}= & \left(z^{1}\right)_{\phi}^{2}+\left(z^{2}\right)_{\phi}^{2}+\left(z^{3}\right)_{\phi}^{2} \\
= & \sum_{i}\left(y_{i}\right)_{\phi}^{2}\left(1+\frac{r^{2}}{3}\left(\frac{R}{2}-\lambda_{i}-\sum_{k} \lambda_{k} y_{k}^{2}\right)\right) \\
& -\frac{r^{2}}{3} \sum_{i} y_{i}\left(y_{i}\right)_{\phi}\left(\sum_{i} \lambda_{i} y_{i}^{2}\right)_{\phi}+O\left(r^{4}\right) \\
= & \sin ^{2} \theta+\frac{r^{2}}{3}\left[\frac{R}{2} \sin ^{2} \theta-\sum_{i} \lambda_{i}\left(\left(y_{i}\right)_{\phi}^{2}+y_{i}^{2} \sin ^{2} \theta\right)\right]+O\left(r^{4}\right) \\
= & \sin ^{2} \theta\left[1+\frac{r^{2}}{3}\left(\frac{R}{2}-\lambda_{1}\left(\sin ^{2} \phi+\cos ^{2} \phi \sin ^{2} \theta\right)\right.\right. \\
& \left.\left.-\lambda_{2}\left(\cos ^{2} \phi+\sin ^{2} \phi \sin ^{2} \theta\right)-\lambda_{3} \cos ^{2} \theta\right)\right]+O\left(r^{4}\right) \\
= & \sin ^{2} \theta\left[1+\frac{r^{2}}{3}\left(\lambda_{12} \sin ^{2} \theta+\lambda_{13} \cos ^{2} \theta \sin ^{2} \phi+\lambda_{23} \cos ^{2} \theta \cos ^{2} \phi\right)\right] \\
& +O\left(r^{4}\right),
\end{aligned}
$$

and

$$
\begin{aligned}
\hat{Z}_{\theta} \cdot \hat{Z}_{\phi}= & \left(z^{1}\right)_{\theta}\left(z^{1}\right)_{\phi}+\left(z^{2}\right)_{\theta}\left(z^{2}\right)_{\phi}+\left(z^{3}\right)_{\theta}\left(z^{3}\right)_{\phi} \\
= & \sum_{i}\left(y^{i}\right)_{\theta}\left(y^{i}\right)_{\phi}+\frac{r^{2}}{3} \sum_{i}\left(y^{i}\right)_{\theta}\left(y^{i}\right)_{\phi}\left(\frac{R}{2}-\lambda_{i}-\sum_{k} \lambda_{k}\left(y^{k}\right)^{2}\right) \\
& -\frac{r^{2}}{6}\left(\sum_{i} y^{i}\left(y^{i}\right)_{\theta}\right)\left(\sum_{i} \lambda_{i}\left(y^{i}\right)^{2}\right)_{\phi}-\frac{r^{2}}{6}\left(\sum_{i} y^{i}\left(y^{i}\right)_{\phi}\right) \\
& \times\left(\sum_{i} \lambda_{i}\left(y^{i}\right)^{2}\right)_{\theta}+O\left(r^{4}\right) \\
= & -\frac{r^{2}}{3} \sum_{i}\left(y^{i}\right)_{\theta}\left(y^{i}\right)_{\phi} \lambda_{i}+O\left(r^{4}\right) \\
= & \frac{r^{2}}{3}\left(-\lambda_{13}+\lambda_{23}\right) \sin \theta \cos \theta \cos \phi \sin \phi+O\left(r^{4}\right) .
\end{aligned}
$$

Thus, we see that

$$
\|\tilde{h}-\hat{h}\|_{C^{3}}=O\left(r^{4}\right) .
$$

This completes the proof of the claim. Next we want to compute $\hat{Z} \cdot \hat{n}$. 
Let $A=\left(y^{1}, y^{2}, y^{3}\right), B=A_{\theta}, C=\frac{1}{\sin \theta} A_{\phi}, \bar{A}=\left(\bar{A}_{1}, \bar{A}_{2}, \bar{A}_{3}\right), \bar{B}=\left(\bar{B}_{1}\right.$, $\left.\bar{B}_{2}, \bar{B}_{3}\right)$ and $\bar{C}=\left(\bar{C}_{1}, \bar{C}_{2}, \bar{C}_{3}\right)$, where

$$
\begin{aligned}
& \bar{A}_{i}=\frac{r^{2} y^{i}}{6}\left(\frac{R}{2}-\lambda_{i}-\sum_{i} \lambda_{k}\left(y^{k}\right)^{2}\right) \\
& \bar{B}_{i}=\frac{\left(y^{i}\right)_{\theta} r^{2}}{6}\left(\frac{R}{2}-\lambda_{i}-\sum_{k} \lambda_{k}\left(y^{k}\right)^{2}\right)-\frac{r^{2}}{6} y^{i}\left(\sum_{k} \lambda_{k}\left(y^{k}\right)^{2}\right)_{\theta} \\
& \bar{C}_{i}=\frac{\left(y^{i}\right)_{\phi} r^{2}}{6 \sin \theta}\left(\frac{R}{2}-\lambda_{i}-\sum_{k} \lambda_{k}\left(y^{k}\right)^{2}\right)-\frac{r^{2}}{6 \sin \theta} y^{i}\left(\sum_{k} \lambda_{k}\left(y^{k}\right)^{2}\right)_{\phi} .
\end{aligned}
$$

Note that $A, B, C$ are orthonormal and positively oriented in $\mathbb{R}^{3}$ for $A \in \mathbb{S}^{2}$. Let $e_{1}=\partial_{\theta}$ and $e_{2}=\frac{1}{\sin \theta} \partial_{\phi}$ as before. Then,

$$
\begin{aligned}
\hat{Z} \cdot \hat{Z}_{1} \wedge \hat{Z}_{2} & =(A+\bar{A}) \cdot(B+\bar{B}) \wedge(C+\bar{C}) \\
& =A \cdot B \wedge C+A \cdot B \wedge \bar{C}+A \cdot \bar{B} \wedge C+\bar{A} \cdot B \wedge C+O\left(r^{4}\right) \\
& =1+C \cdot \bar{C}+B \cdot \bar{B}+A \cdot \bar{A}+O\left(r^{4}\right) .
\end{aligned}
$$

Now

$$
\begin{aligned}
A \cdot \bar{A}= & \frac{r^{2}}{6}\left(\frac{R}{2}-2 \sum_{k} \lambda_{k}\left(y^{k}\right)^{2}\right) \\
B \cdot \bar{B}= & \frac{r^{2}}{6}\left(\frac{R}{2}-\sum_{k} \lambda_{k}\left(\left(y^{k}\right)_{\theta}^{2}+\left(y^{k}\right)^{2}\right)\right) \\
= & \frac{r^{2}}{6}\left(\frac{R}{2}-\left(\lambda_{1} \cos ^{2} \phi+\lambda_{2} \sin ^{2} \phi+\lambda_{3}\right)\right) \cdot \\
C \cdot \bar{C}= & \frac{r^{2}}{6 \sin ^{2} \theta}\left(\frac{R}{2} \sin ^{2} \theta-\sum_{k} \lambda_{k}\left(\left(y^{k}\right)_{\phi}^{2}+\left(y^{k}\right)^{2} \sin ^{2} \theta\right)\right) \\
= & \frac{r^{2}}{6}\left(\frac{R}{2}-\left(\lambda_{1}\left(\sin ^{2} \phi+\sin ^{2} \theta \cos ^{2} \phi\right)\right.\right. \\
& \left.\left.+\lambda_{2}\left(\cos ^{2} \phi+\sin ^{2} \theta \sin ^{2} \phi\right)+\lambda_{3} \cos ^{2} \theta\right)\right) .
\end{aligned}
$$

So

$$
\hat{Z} \cdot \hat{Z}_{1} \wedge \hat{Z}_{2}=1+\frac{r^{2}}{6}\left(\frac{R}{2}-3 \sum \lambda_{k}\left(y^{k}\right)^{2}\right)+O\left(r^{4}\right)
$$


Noting that

$$
\begin{aligned}
\left|\hat{Z}_{1} \wedge \hat{Z}_{2}\right|^{2}= & 1+\frac{r^{2}}{3}\left[\left(\lambda_{13}\left(\cos ^{2} \phi+\cos ^{2} \theta \sin ^{2} \phi\right)+\lambda_{12} \sin ^{2} \theta\right.\right. \\
& \left.\left.+\lambda_{23}\left(\sin ^{2} \phi+\cos ^{2} \theta \cos ^{2} \phi\right)\right)\right]+O\left(r^{4}\right) \\
= & 1-\frac{r^{2}}{3} \sum \lambda_{k}\left(y^{k}\right)^{2}+O\left(r^{4}\right)
\end{aligned}
$$

we have

$$
\left|\hat{Z}_{1} \wedge \hat{Z}_{2}\right|^{-1}=1+\frac{r^{2}}{6} \sum \lambda_{k}\left(y^{k}\right)^{2}+O\left(r^{4}\right)
$$

Combining (3.30) and (3.31)

$$
\hat{Z} \cdot \hat{n}=\frac{\hat{Z} \cdot \hat{Z}_{1} \wedge \hat{Z}_{2}}{\left|\hat{Z}_{1} \wedge \hat{Z}_{2}\right|}=1+\frac{r^{2}}{6}\left(\frac{R}{2}-2 \sum \lambda_{k}\left(y^{k}\right)^{2}\right)+O\left(r^{4}\right)
$$

This completes the proof of the lemma.

Lemma 3.4. Let $K$ and $H$ be the Gauss curvature and the mean curvature of $S_{r}$ in $g$ and $H_{0}$ be the mean curvature of $\left(S_{r},\left.g\right|_{S_{r}}\right)$ when embedded in $\mathbb{R}^{3}$. Then,

$$
\begin{aligned}
K & =\frac{1}{r^{2}}+\frac{R}{2}-\frac{4}{3} R_{i j} \frac{x^{i} x^{j}}{r^{2}}+O(r), \\
H & =\frac{2}{r}-\frac{1}{3} R_{i j} \frac{x^{i} x^{j}}{r}+O\left(r^{2}\right)
\end{aligned}
$$

and

$$
H_{0}=\frac{2}{r}+r\left(\frac{R}{2}-\frac{4}{3} R_{i j} \frac{x^{i} x^{j}}{r^{2}}\right)+O\left(r^{2}\right) .
$$

Proof. We continue to use the normal coordinates as in Lemma 3.1. Then $n=\frac{\partial}{\partial r}$ is the outward normal of $S_{r}$. Let $h_{i j}=g_{i j}-n_{i} n_{j}$ be the induced metric on $S_{r}$ with $n_{i}=\frac{x^{i}}{r}$. By Lemma 3.1, the Christoffel symbols are given by:

$$
\Gamma_{i j}^{k}=\frac{1}{3}\left(R_{k i m j}+R_{k j m i}\right) x^{m}+O\left(r^{2}\right) .
$$


where the curvature is evaluated at $p$. Since $\nabla_{n} n=0$, the second fundamental form $A$ in these coordinates is given by

$$
\begin{aligned}
A_{i j} & =n_{j ; i} \\
& =\frac{\partial n_{j}}{\partial x^{i}}-\Gamma_{i j}^{k} n_{k} \\
& =\frac{\delta_{i j}}{r}-\frac{x^{i} x^{j}}{r^{3}}-\frac{2}{3} R_{k i m j} \frac{x^{k} x^{m}}{r}+O\left(r^{2}\right) .
\end{aligned}
$$

Let $\left\{e_{1}, e_{2}\right\}$ be an orthonormal frame with respect to the Euclidean metric on $S_{r}$ and let $\lambda_{1}$ and $\lambda_{2}$ be the eigenvalues of $A$. Then

$$
\begin{aligned}
\lambda_{1} \lambda_{2}= & \frac{A\left(e_{1}, e_{1}\right) A\left(e_{2}, e_{2}\right)-A^{2}\left(e_{1}, e_{2}\right)}{g\left(e_{1}, e_{2}\right) g\left(e_{2}, e_{2}\right)-g^{2}\left(e_{1}, e_{2}\right)} \\
= & \left(\frac{1}{r^{2}}-\frac{2}{3} R_{k i m j} \frac{x^{k} x^{m}}{r^{2}}\left(e_{1}\left(x^{i}\right) e_{1}\left(x^{j}\right)+e_{2}\left(x^{i}\right) e_{2}\left(x^{j}\right)\right)+O(r)\right) \\
& \times\left(1-\frac{1}{3} R_{i k m j} x^{k} x^{m}\left(e_{1}\left(x^{i}\right) e_{1}\left(x^{j}\right)+e_{2}\left(x^{i}\right) e_{2}\left(x^{j}\right)\right)+O\left(r^{3}\right)\right) \\
= & \frac{1}{r^{2}}-\frac{1}{3} R_{k i m j} \frac{x^{k} x^{m}}{r^{2}}\left(e_{1}\left(x^{i}\right) e_{1}\left(x^{j}\right)+e_{2}\left(x^{i}\right) e_{2}\left(x^{j}\right)\right)+O(r) \\
= & \frac{1}{r^{2}}-\frac{1}{3} R_{k m} \frac{x^{k} x^{m}}{r^{2}}+O(r),
\end{aligned}
$$

where we have used the fact that $\sum_{i}\left(e_{a}\left(x^{i}\right)\right)^{2}=1$ and $e_{a}\left(\sum_{i}\left(x^{i}\right)^{2}\right)=0$ on $S_{r}$ for $a=1,2$, and the fact that

$$
e_{1}\left(x^{i}\right) e_{1}\left(x^{j}\right)+e_{2}\left(x^{i}\right) e_{2}\left(x^{j}\right)=\delta_{i j}-\frac{x^{i} x^{j}}{r^{2}}
$$

Hence by the Gauss equation, for $x \in S_{r}$,

$$
\begin{aligned}
K(x) & =\lambda_{1} \lambda_{2}+\frac{1}{2} h^{i k} h^{j l} R_{i j k l}(x) \\
& =\frac{1}{r^{2}}-\frac{1}{3} R_{k m} \frac{x^{k} x^{m}}{r^{2}}+\frac{1}{2} h^{i k} h^{j l} R_{i j k l}(p)+O(r) \\
& =\frac{1}{r^{2}}+\frac{1}{2} R(p)-\frac{4}{3} R_{i j}(p) \frac{x^{i} x^{j}}{r^{2}}+O(r),
\end{aligned}
$$


where $h^{i j}=g^{i j}-n^{i} n^{j}$. On the other hand, for $x \in S_{r}$,

$$
\begin{aligned}
H(x) & =h^{i j} A_{i j} \\
& =h^{i j}\left(\frac{\delta_{i j}}{r}-\frac{2}{3} R_{k i m j} \frac{x^{k} x^{m}}{r}\right)+O\left(r^{2}\right) \\
& =\left(\delta_{i j}-\frac{1}{3} R_{i k m j} x^{k} x^{m}-\frac{x^{i} x^{j}}{r^{2}}\right)\left(\frac{\delta_{i j}}{r}-\frac{2}{3} R_{k i m j} \frac{x^{k} x^{m}}{r}\right)+O\left(r^{2}\right) \\
& =\frac{2}{r}-\frac{1}{3} R_{i j} \frac{x^{i} x^{j}}{r}+O\left(r^{2}\right),
\end{aligned}
$$

where we have used the fact that $h^{i j} x^{i} x^{j}=0$.

It remains to prove the last assertion. Let $Z_{r}$ be the embedding as in the proof of Lemma 3.3. One may conclude that by an isometry of $\mathbb{R}^{3}$, we have $\left\|Z_{r}-\mathrm{Id}\right\|_{C^{2}\left(\mathbb{S}^{2}\right)}=O\left(r^{2}\right)$, where Id is the identity map of $\mathbb{S}^{2}$. Let $H_{r}$ and $K_{r}$ be the mean curvature and the Gauss curvature of $Z_{r}\left(\mathbb{S}^{2}\right)$. Let $\left\{e_{1}, e_{2}\right\}$ be an orthonormal frames on $\mathbb{S}^{2}$ with respect to the standard metric, then the metric tensor $h$ and the second fundamental form $A$ of the surface $Z_{r}\left(\mathbb{S}^{2}\right)$ satisfy:

$$
h\left(e_{a}, e_{b}\right)=\delta_{a b}+\alpha_{a b}, \quad A\left(e_{a}, e_{b}\right)=\delta_{a b}+\beta_{a b},
$$

where $\alpha_{a b}=O\left(r^{2}\right)$ and $\beta_{a b}=O\left(r^{2}\right)$. Hence, we have

$$
\begin{aligned}
& K_{r}=1-\alpha_{11}-\alpha_{22}+\beta_{11}+\beta_{22}+O\left(r^{4}\right), \\
& H_{r}=2-\alpha_{11}-\alpha_{22}+\beta_{11}+\beta_{22}+O\left(r^{4}\right) .
\end{aligned}
$$

After rescale to an embedding of $\left(S_{r},\left.g\right|_{S_{r}}\right)$ in $\mathbb{R}^{3}$, we conclude that

$$
K=\frac{1}{r^{2}}\left(1-\alpha_{11}-\alpha_{22}+\beta_{11}+\beta_{22}\right)+O\left(r^{2}\right)
$$

and

$$
H_{0}=\frac{1}{r}\left(2-\alpha_{11}-\alpha_{22}+\beta_{11}+\beta_{22}\right)+O\left(r^{3}\right)
$$

From these and (3.33), (3.35) follows.

We are ready to prove the following (Theorem 1.3): 
Theorem 3.1. Let $(N, g)$ be a Riemannian manifold of dimension $3, p$ be a fixed interior point on $N$ and $S_{r}$ be the geodesic sphere of radius $r$ center at $p$. For $r$ small enough, we have

$$
m_{\mathrm{BY}}\left(S_{r}\right)=\frac{r^{3}}{12} R(p)+\frac{r^{5}}{1440}\left[24|\operatorname{Ric}|^{2}(p)-13 R^{2}(p)+12 \Delta R(p)\right]+O\left(r^{6}\right)
$$

here $\Delta$ is Laplacian operator of $(M, g)$.

Proof. For $r$ small, let $Z$ be the isometric embedding of $\left(S_{r},\left.g\right|_{S_{r}}\right)$ in $\mathbb{R}^{3}$ as in Lemma 3.3 and let $H_{0}$ be the mean curvature of $Z\left(S_{r}\right)$ in $\mathbb{R}^{3}$. Let

$$
k_{0}=\frac{R}{2}-\frac{4}{3} R_{i j} \frac{x^{i} x^{j}}{r^{2}}, \quad h_{1}=r k_{0}, \quad n_{3}=\frac{r^{3}}{6}\left(\frac{R}{2}-2 R_{i j} \frac{x^{i} x^{j}}{r^{2}}\right) .
$$

By Lemmas 3.4 and 3.3, we have

$$
K=\frac{1}{r^{2}}+k_{0}+O(r), \quad H_{0}=\frac{2}{r}+h_{1}+O\left(r^{2}\right), \quad Z \cdot n=r+n_{3}+O\left(r^{4}\right) .
$$

As in the proof of Theorem 2.1 in Section 2, by one of the Minkowski integral formulae [16, Lemma 6.2.9] and Lemma 3.4, we have

$$
\begin{aligned}
\int_{S_{r}} H_{0} d \Sigma_{r} & =2 \int_{S_{r}} K Z \cdot n d \Sigma_{r} \\
& =\frac{1}{r^{2}} \int_{S_{r}} Z \cdot n d \Sigma_{r}+2 \int_{S_{r}}\left(K-\frac{1}{r^{2}}\right)\left(r+n_{3}\right) d \Sigma_{r}+O\left(r^{6}\right) \\
& =6 r^{-2} V_{0}(r)+2 r \int_{S_{r}}\left(K-\frac{1}{r^{2}}\right) d \Sigma_{r}+2 \int_{S_{r}} k_{0} n_{3} d \Sigma_{r}+O\left(r^{6}\right) \\
& =6 r^{-2} V_{0}(r)+8 \pi r-\frac{2 \mathcal{A}(r)}{r}+2 \int_{S_{r}} k_{0} n_{3} d \Sigma_{r}+O\left(r^{6}\right)
\end{aligned}
$$

where $V_{0}(r)$ is the volume inside $Z\left(S_{r}\right)$ in $\mathbb{R}^{3}$. 
By another Minkowski integral formula, we obtain

$$
\begin{aligned}
2 \mathcal{A}(r) & =\int_{S_{r}} H_{0} Z \cdot n d \Sigma_{r} \\
& =\int_{S_{r}} \frac{2}{r} Z \cdot n d \Sigma_{r}+\int_{S_{r}}\left(H_{0}-\frac{2}{r}\right)\left(r+n_{3}\right) d \Sigma_{r}+O\left(r^{7}\right) \\
& =6 r^{-1} V_{0}(r)+r \int_{S_{r}} H_{0} d \Sigma_{r}-2 \mathcal{A}(r)+\int_{S_{r}} h_{1} n_{3} d \Sigma_{r}+O\left(r^{7}\right) \\
& =6 r^{-2} V_{0}(r)+r \int_{S_{r}} H_{0} d \Sigma_{r}-2 \mathcal{A}(r)+r \int_{S_{r}} k_{0} n_{3} d \Sigma_{r}+O\left(r^{7}\right) .
\end{aligned}
$$

Hence,

$$
\int_{S_{r}} H_{0} d \Sigma_{r}=-6 r^{-2} V_{0}(r)+4 r^{-1} \mathcal{A}(r)-\int_{S_{r}} k_{0} n_{3} d \Sigma_{r}+O\left(r^{6}\right) .
$$

By (3.43) and (3.45), we have

$$
\begin{aligned}
\int_{S_{r}} H_{0} d \Sigma_{r} & =4 \pi r+r^{-1} \mathcal{A}(r)+\int_{S_{r}} k_{0} n_{3} d \Sigma_{r}-\frac{1}{2} \int_{S_{r}} k_{0} n_{3} d \Sigma_{r}+O\left(r^{6}\right) \\
& =8 \pi r+\frac{A_{4}+A_{6}}{r}+\frac{1}{2} \int_{S_{r}} k_{0} n_{3} d \Sigma_{r}+O\left(r^{6}\right)
\end{aligned}
$$

where we have used Lemma 3.2. Combining this with Lemma 3.1, we have

$$
\int_{S_{r}}\left(H_{0}-H\right) d \Sigma_{r}=-\frac{3 A_{4}+5 A_{6}}{r}+\frac{1}{2} \int_{S_{r}} k_{0} n_{3} d \Sigma_{r}+O\left(r^{6}\right) .
$$

Now by (3.7) and (3.11)

$$
\begin{aligned}
\int_{S_{r}} k_{0} n_{3} d \Sigma_{r} & =\frac{r^{3}}{6} \int_{S_{r}}\left(\frac{R}{2}-\frac{4}{3} R_{i j} \frac{x^{i} x^{j}}{r^{2}}\right)\left(\frac{R}{2}-2 R_{i j} \frac{x^{i} x^{j}}{r^{2}}\right) d \Sigma_{r} \\
& =\frac{r^{3}}{6} \int_{S_{r}}\left(\frac{R}{2}-\frac{4}{3} R_{i j} \frac{x^{i} x^{j}}{r^{2}}\right)\left(\frac{R}{2}-2 R_{i j} \frac{x^{i} x^{j}}{r^{2}}\right) d \Sigma_{r}^{0}+O\left(r^{6}\right) \\
& =\frac{\pi r^{5}}{270}\left(64|\mathrm{Ric}|^{2}-23 R^{2}\right) .
\end{aligned}
$$

The theorem follows from $(3.47),(3.48)$ and Lemma 3.2.

As a corollary, we have 
Corollary 3.2. With the assumptions and notation as in Theorem 3.1, suppose $R \geq 0$ in a neighborhood of $p$, then

$$
\lim _{r \rightarrow 0} \frac{m_{\mathrm{BY}}\left(S_{r}\right)}{r^{5}} \geq 0
$$

Equality holds if and only if $(N, g)$ is flat at $p$ and $R$ vanishes up to second order at $p$.

Proof. By the result of [23] on the positivity of the Brown-York mass, we know that (3.49) is true. However, in this special case, one can deduce this from the theorem. In fact, if $R(p)>0$, then by (3.42), we have

$$
\lim _{r \rightarrow 0} \frac{m_{\mathrm{BY}}\left(S_{r}\right)}{r^{5}}=\infty>0
$$

In case $R(p)=0$, then $R(p)$ is a minimum of $R$ because $R \geq 0$. It is easy to see that (3.49) is still true.

It is obvious that if $(N, g)$ is flat at $p$ and $R$ vanishes up to second order at $p$, then equality holds in (3.49). Conversely, if the equality holds in (3.49), then we must have $R(p)=0, \nabla R(p)=0, \Delta R(p)=0$ and $|\operatorname{Ric}|(p)=0$. Since $R$ has a minimum at $p$, the Hessian of $R$ has non-negative eigenvalues. So the Hessian of $R$ must be zero at $p$ because $\Delta R(p)=0$. Moreover, since $N$ has dimension $3,|\operatorname{Ric}|(p)=0$ implies that $(N, g)$ is flat at $p$.

Remark 3.1. From the proof, it is easy to see that (3.49) is true if $R(p)=0$ and $\Delta R(p) \geq 0$ and the equality holds only if $g$ is flat at $p$.

One should compare the corollary to the following fact: If $M$ is an AF manifold with non-negative scalar curvature, suppose the Brown-York mass of the coordinate spheres converge to zero, then $M$ must be the Euclidean space. This follows from Theorem 2.1 and the positive mass theorem in Schoen-Yau [21] and Witten [24].

For the expansion of the Hawking mass, we have:

Theorem 3.2. With the same notations and assumptions in Theorem 3.1, we have

$$
m_{\mathrm{H}}\left(S_{r}\right)=\frac{r^{3}}{12} R(p)+\frac{r^{5}}{720}\left(6 \Delta R(p)-5 R^{2}(p)\right)+O\left(r^{6}\right) .
$$


Proof. By Lemma 3.4, we have

$$
H=\frac{2}{r}+H_{1}+O\left(r^{2}\right),
$$

where $H_{1}=-\frac{1}{3 r} R_{i j} x^{i} x^{j}$. Hence

$$
H^{2}=-4 r^{-2}+4 r^{-1} H+H_{1}^{2}+O\left(r^{3}\right) .
$$

Then

$$
\begin{aligned}
\int_{S_{r}} H^{2} d \Sigma_{r}= & -\frac{4 \mathcal{A}(r)}{r^{2}}+\frac{4}{r} \int_{S_{r}} H d \Sigma_{r}+\int_{S_{r}} H_{1}^{2} d \Sigma_{r}^{0}+O\left(r^{5}\right) \\
= & -\frac{4\left(4 \pi r^{2}+A_{4}+A_{6}\right)}{r^{2}}+\frac{4}{r} \cdot\left(8 \pi r+\frac{4 A_{4}}{r}+\frac{6 A_{6}}{r}\right) \\
& +\int_{S_{r}} H_{1}^{2} d \Sigma_{r}^{0}+O\left(r^{5}\right) \\
= & 16 \pi+\frac{12 A_{4}}{r^{2}}+\frac{20 A_{6}}{r^{2}}+\int_{S_{r}} H_{1}^{2} d \Sigma_{r}^{0}+O\left(r^{5}\right)
\end{aligned}
$$

Hence,

$$
16 \pi-\int_{S_{r}} H^{2} d \Sigma_{r}=-\frac{12 A_{4}}{r^{2}}-\frac{20 A_{6}}{r^{2}}-\int_{S_{r}} H_{1}^{2} d \Sigma_{r}^{0}+O\left(r^{5}\right) .
$$

On the other hand,

$$
\begin{aligned}
\frac{\mathcal{A}^{1 / 2}(r)}{(16 \pi)^{3 / 2}} & =\frac{2 \pi^{1 / 2} r}{(16 \pi)^{3 / 2}}\left(1+\frac{A_{4}}{8 \pi r^{2}}+O\left(r^{4}\right)\right) \\
& =\frac{r}{32 \pi}\left(1+\frac{A_{4}}{8 \pi r^{2}}+O\left(r^{4}\right)\right) .
\end{aligned}
$$

So

$$
m_{\mathrm{H}}\left(S_{r}\right)=-\frac{3 A_{4}}{8 \pi r}-\frac{5 A_{6}}{8 \pi r}-\frac{r}{32 \pi} \int_{S_{r}} H_{1}^{2} d \Sigma_{r}^{0}-\frac{3 A_{4}^{2}}{64 \pi^{2} r^{3}}+O\left(r^{6}\right) .
$$

By (3.11) and Lemma 3.2, the result follows.

Hence, the expansions of the Brown-York mass and the Hawking mass are equal up to order $r^{3}$. However, they differ on the term of order $r^{5}$.

As in the case of large-sphere limit, we can compare $V(r)$ and $V_{0}(r)$, where $V(r)$ is the volume of the geodesic ball of radius $r$ at $p$ and $V_{0}(r)$ is the volume of the region bounded by $S_{r}$ when embedded in $\mathbb{R}^{3}$. 
Theorem 3.3. With the above notations, we have

$$
V_{0}(r)-V(r)=-\frac{\pi}{15} R r^{5}+\frac{\pi r^{7}}{5670}\left(173 R^{2}-454|\operatorname{Ric}|^{2}-27 \Delta R(p)\right)+O\left(r^{8}\right) .
$$

Proof. By (3.43) and (3.45)

$$
\int_{S_{r}} H_{0} d \Sigma_{r}=6 r^{-2} V_{0}(r)+8 \pi r-\frac{2 \mathcal{A}(r)}{r}+2 \int_{S_{r}} k_{0} n_{3} d \Sigma_{r}+O\left(r^{6}\right),
$$

and

$$
\int_{S_{r}} H_{0} d \Sigma_{r}=-6 r^{-2} V_{0}(r)+4 r^{-1} \mathcal{A}(r)-\int_{S_{r}} k_{0} n_{3} d \Sigma_{r}
$$

where $k_{0}$ is as in (3.43).

We have

$$
\begin{aligned}
V_{0}(r) & =\frac{r}{2} \mathcal{A}(r)-\frac{r^{2}}{4} \int_{S_{r}} k_{0} n_{3} d \Sigma_{r}^{0}-\frac{2}{3} \pi r^{3}+O\left(r^{8}\right) \\
& =\frac{4}{3} \pi r^{3}+\frac{r}{2} A_{4}+\frac{r}{2} A_{6}-\frac{r^{2}}{4} \int_{S_{r}} k_{0} n_{3} d \Sigma_{r}^{0}+O\left(r^{8}\right) .
\end{aligned}
$$

On the other hand,

$$
\begin{aligned}
V(r) & =\int_{0}^{r} \mathcal{A}(t) d t \\
& =\frac{4}{3} \pi r^{3}+\int_{0}^{r} A_{4} d t+\int_{0}^{r} A_{6} d t+O\left(r^{8}\right) \\
& =\frac{4}{3} \pi r^{3}+\frac{r}{5} A_{4}+\frac{r}{7} A_{6}+O\left(r^{8}\right) .
\end{aligned}
$$

Hence,

$$
V_{0}(r)-V(r)=\frac{3}{10} r A_{4}+\frac{5}{14} r A_{6}-\frac{r^{2}}{4} \int_{S_{r}} k_{0} n_{3} d \Sigma_{r}^{0}+O\left(r^{8}\right)
$$

By (3.48) and Lemma 3.2, the result follows.

By Theorem 3.3, we see that if scalar curvature is positive at $p$, then $V_{0}(r)<V(r)$, for sufficiently small $r$. More precisely, 
Corollary 3.3. With the assumptions and notations as in Theorem 3.3, suppose $R \geq 0$ in a neighborhood of $p$, then

$$
\lim _{r \rightarrow 0} \frac{V_{0}(r)-V(r)}{r^{7}} \leq 0 .
$$

Equality holds if and only if $(N, g)$ is flat at $p$ and $R$ vanishes up to second order at $p$.

Proof. Similar to the argument of Corollary 3.2, one can derive the result from Theorem 3.3.

\section{Acknowledgments}

Research partially supported by 973 Program (2006CB805905) of China and Fok YingTong Education Foundation (Y.S.). Research partially supported by Earmarked Grant of Hong Kong no. CUHK403005 (L.-F.T.).

\section{References}

[1] R. Arnowitt, S. Deser and C.W. Misner, Coordinate invariance and energy expressions in general relativity, Phys. Rev. (2) 122 (1961), 9971006.

[2] R. Bartnik, The mass of an asymptotically flat manifold, Comm. Pure Appl. Math. 39 (5) (1986), 661-693.

[3] D. Baskaran, S.R. Lau and A.N. Petrov, Center of mass integral in canonical general relativity, Ann. Phys. 307 (1) (2003), 90-131.

[4] H.W. Braden, J.D. Brown, B.F. Whiting and J.W. York, Charged black hole in a grand canonical ensemble, Phys. Rev. D (3) 42 (10) (1990), 3376-3385.

[5] J.D. Brown, S.R. Lau and J.W. York, Canonical quasilocal energy and small spheres, Phys. Rev. D (3) 59 (6) (1999), 064028.

[6] J.D. Brown and J.W. York, Quasilocal energy in general relativity, Mathematical aspects of classical field theory (Seattle, WA, 1991), Contemp. Math. 132, Amer. Math. Soc., Providence, RI, 1992, 129-142.

[7] J.D. Brown and J.W. York, Quasilocal energy and conserved charges derived from the gravitational action, Phys. Rev. D (3) 47 (4) (1993), 1407-1419. 
[8] S. Cohn-Vossen, Zwei Sätze über die Starrheit der Eiflächen, Nachr. Ges. Wiss. Gottingen Math. Phys. Kl., 27 (1927), 125-134.

[9] S.R. Douglas, Review of the definitions of the Bel and Bel-Robinson tensors, Gen. Relativity Gravitation 35 (9) (2003), 1691-1697.

[10] R.S. Hamilton, Three-manifolds with positive Ricci curvature, J. Diff. Geom. 17(2) (1982), 255-306.

[11] S.W. Hawking, Gravitational radiation in an expanding universe, J. Math. Phys. 9 (1968), 598-604.

[12] S.W. Hawking and G.T. Horowitz, The gravitational Hamiltonian, action, entropy and surface terms, Classical Quantum Gravity 13(6) (1996), 1487-1498.

[13] G. Herglotz, Über die Steinersche Formel für Parallelflächen, Abh. Math. Sem. Hansischen Univ. 15 (1943), 165-177.

[14] G. Huisken, An isoperimetric concept for mass and quasilocal mass, in Mathematical Aspects of General Relativity, Report no. 2/2006, Mathematisches Forschungsinstitut Oberwolfach, http://www.mfo.de/ programme/schedule/2006/02/OWR_2006_02.pdf

[15] G. Huisken and T. Ilmanen, The inverse mean curvature flow and the Riemannian Penrose inequality, J. Diff. Geom. 59(3), (2001), 353-437.

[16] W. Klingenberg, A course in differential geometry, Translated from the German by David Hoffman. Graduate Texts in Mathematics 51, Springer-Verlag, New York-Heidelberg, 1978.

[17] P. Miao, Private communication.

[18] L. Nirenberg, The Weyl and Minkowski problems in differential geometry in the large, Comm. Pure Appl. Math. 6 (1953), 337-394.

[19] A.V. Pogorelov, Extrinsic geometry of convex surfaces, Translated from the Russian by Israel Program for Scientific Translations. Translations of Mathematical Monographs 35, American Mathematical Society, Providence, RI, 1973.

[20] R. Sacksteder, The rigidity of hypersurfaces, J. Math. Mech. 11 (1962), 929-939.

[21] R. Schoen and S.-T. Yau, On the proof of the positive mass conjecture in general relativity, Comm. Math. Phys. 65 (1979), 45-76. 
[22] — Lectures on differential geometry, International Press, Cambridge, MA, 1994.

[23] Y.-G. Shi and L.-F. Tam, Positive mass theorem and the boundary behaviors of compact manifolds with non-negative scalar curvature, J. Diff. Geom. 62 (2002), 79-125.

[24] E. Witten, A new proof of the positive energy theorem, Comm. Math. Phys. 80 (1981), 381-402.

Department of Mathematics

JINAN UNIVERSITY

GuANGZHOU 510632

P.R. CHINA

E-mail address: txqfan@jnu.edu.cn

Key Laboratory of Pure and Applied mathematics

School of Mathematics Science

PEKING UNIVERSITY

BEIJING 100871

P.R. CHINA

E-mail address: ygshi@math.pku.edu.cn

The Institute of Mathematical Sciences and Department of MATHEMATiCs

The Chinese University of Hong Kong

SHATIN

Hong Kong, China

E-mail address: lftam@math.cuhk.edu.hk

Received November 18, 2007 